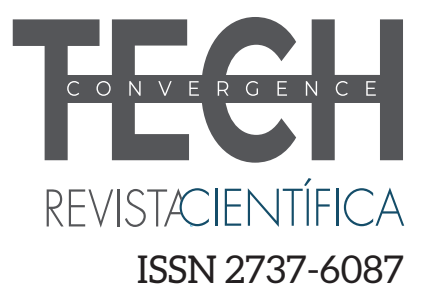

\title{
IMPLEMENTACIÓN DE LA ASIGNATURA DE APRENDER A APRENDER COMO PARTE DE LA INNOVACIÓN EDUCATIVA EN EL MARCO DE LA REFORMA DE LICENCIATURA DE LA UAEH
}

M.C.E. Catalina Polo Jiménez ${ }^{1}$

Colaboración: Mtro. Othón Straffon Muris, Lic. Juventino Ortega Canales

${ }^{1}$ Universidad Autónoma del Estado de Hidalgo. Instituto de Ciencias Básicas e Ingeniería. Profesor Investigador y docente de la asignatura de Aprender a Aprender

E-mail: katypolo770@gmail.com (iD https://orcid.org/0000-0001-7163-2134
Fecha de recepción: 05/08/2020

Fecha de resultado: 05/02/2021

\section{RESUMEN}

A partir de la Reforma educativa llevada a cabo en la Universidad Autónoma del Estado de Hidalgo y para fortalecer la formación integral del estudiante se diseñan las asignaturas institucionales, una de las cuales es Aprender a Aprender. En el 2013 el Instituto de Ciencias Básicas e Ingeniería inicia con la implementación de cinco programas educativos aprobados por el $\mathrm{H}$. Consejo Universitario. En este contexto, esta asignatura inicia con el trabajo académico, las actividades y trabajo colegiado de la academia permiten generar propuestas innovadoras para fortalecer el desarrollo académico y personal de

\section{ABSTRACT}

From the Educational Reform carried out at the Autonomous University of the State of Hidalgo and to strengthen the integral training of the student are designed the institutional subjects, one of which is Learn to Learn. In 2013 the Institute of Basic Sciences and Engineering begins with the implementation of five educational programs approved by the Brother University Council. In this context, this subject begins with academic work, the activities and collegiate work of the academy allow to generate innovative proposals to strengthen the academic and los alumnos, como el Encuentro de Habilidades de la Comunicación, Talleres del Estudiante estratégico, Reconciliándome con mi cerebro y Habilidades de autorregulación y metacognición, Diseño de Materiales multimedia para elaborar el Perfil del Estudiante e implementación del Aprendizaje Cooperativo como estrategia de enseñanza para fortalecer habilidades de liderazgo, trabajo en equipo y desarrollo de hábitos de estudio son algunas de las acciones que han marcado el cambio y sigue siendo en la actualidad una asignatura esencial en el ámbito universitario.

Palabras Clave: aprender a aprender, formación integral del estudiante

personal development of students, such as the Meeting of Communication Skills, Workshops of the Strategic Student, Reconciling with my brain and Skills of self-regulation and metacognition, Design of Multimedia Materials to develop the Student Profile and implementation of Cooperative Learning as a teaching strategy to strengthen leadership skills, teamwork and development of study habits are some of the actions that have marked change and remains today an essential subject in the university field.

Keywords: learn to learn, comprehensive student training 
Forma sugerida de citar: Forma sugerida de citar: Polo Jiménez, C. (2021). Implementación de la asignatura de aprender a aprender como parte de la innovación educativa en el marco de la reforma de licenciatura de la UAEH. Convergence Tech Revista Científica. 5(1), 6-17. https://doi.org/10.53592/convtech.v5iV.22

$\mathbf{E}$ n el marco de la reforma curricular que se llevó a cabo en la Universidad Autónoma del Estado de Hidalgo a partir del 2007 que se aplicó a los 43 programas de licenciatura y 5 de nueva creación y atendiendo a políticas educativas emitidas a los gobiernos latinoamericanos por organismos como: el Banco Mundial, el Fondo Monetario Internacional, el Banco Interamericano de Desarrollo y la Organización para la Cooperación y el
Desarrollo Económico con proyección a la incorporación de estos países, para mejorar las condiciones económicas y culturales. Con el fin de implementar dichas reformas en la institución, se llevó a cabo el diseño de un Modelo Curricular Integral para la reforma de licenciatura. (Cuevas, W2010)

El Modelo curricular integral de nivel licenciatura se conforma con tres dimensiones:

\section{Ilustración 1 » Dimensiones del Modelo Curricular Integral de nivel licenciatura de la UAEH.}

\begin{tabular}{|l|l|}
\hline $\begin{array}{c}\text { Dimensión } \\
\text { socio-cultural }\end{array}$ & $\begin{array}{l}\text { Brinda la preparación para vivir en un mundo caracterizado por la evolución de las técnicas, el } \\
\text { desarrollo de la información y medios de comunicación. }\end{array}$ \\
\hline $\begin{array}{c}\text { Didáctico - } \\
\text { pedagógica }\end{array}$ & Define los factores psicopedagógicos para determinar los principios sobre la enseñanza-aprendizaje. \\
\hline $\begin{array}{c}\text { Epistemológica - } \\
\text { profesional }\end{array}$ & $\begin{array}{l}\text { Se enfoca en las actualizaciones, exigencias científicas, filosóficas, así como las competencias } \\
\text { profesionales que demanda el entorno. }\end{array}$ \\
\hline
\end{tabular}

Y a partir de la dimensión sociocultural surgen los Ejes transversales para hacer frente a las características deseables en los profesionistas y se conceptualizan como conocimientos generales de carácter universal que serán transmitidos de manera transversal a todos los estudiantes durante su formación profesional:

Ilustración 2 » Ejes transversales a partir de la Dimensión sociocultural. MCI. UAEH.

Educación

ambiental

Promueve el respeto y

cuidado del medio ambiente.

\section{Educación para}

la equidad

Propicia la valoración y respeto por la interculturalidad y garantiza la igualdad de oportunidades.

A partir de los ejes transversales se generan las asignaturas institucionales que apoyaran la implementación

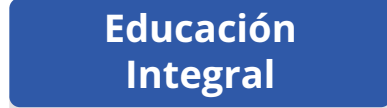

Desarrolla la personalidad del ser humano en forma equilibrada.

Ser biopsicosocial.
Educación para la vida activa

Desarrolla capacidades emprendedoras y creativas para identificar, plantear y resolver problemas. de dichos ejes además de contribuir con el logro de los niveles de competencias profesionales:

Ilustración 3 » Asignatura institucionales a partir de los Ejes transversales. ICBI. UAEH
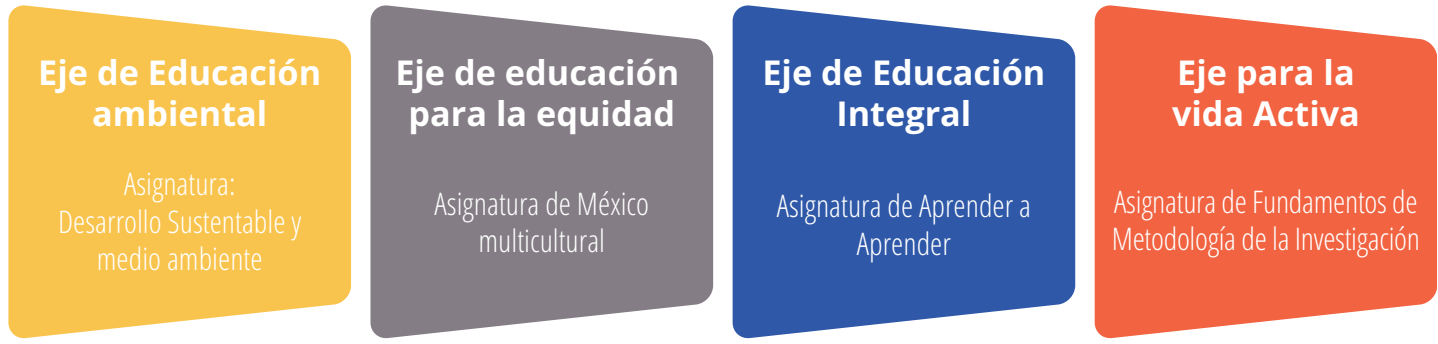

Implementación de la asignatura de aprender a aprender como parte de la innovación educativa en el marco de la reforma de licenciatura de la UAEH

SEPTIEMBRE-FEBRERO (2021) VOL. 5, NO. 1,6-17

https://doi.org/10.53592/convtech.v5iV.22 


\section{Asignatura de Aprender a Aprender}

En el semestre enero - junio del 2013, en el Instituto de ciencias básicas e ingeniería se implementan los siguientes programas educativos aprobados por el $\mathrm{H}$. Consejo Universitario que fueron rediseñados conforme al Modelo Educativo y Modelo Curricular Integral de nivel licenciatura e ingresa la primer generación:

- Licenciatura en Ingeniería Minero Metalúrgica

- Licenciatura en Ciencias computacionales

- Licenciatura en Ingeniería Civil
- Licenciatura en Ingeniería Industrial

- Licenciatura en Biología

La asignatura de Aprender a aprender quedó diseñada en su primer versión en diciembre del 2010 cuyo objetivo y estructura temática fue la siguiente:

Desarrollar las habilidades de comunicación y estrategias de aprendizaje a través de la aplicación de técnicas y procedimientos para fomentar el desarrollo personal y académico.

Ilustración 4» Estructura temática de la asignatura de Aprender a Aprender de la UAEH. Versión 2010.

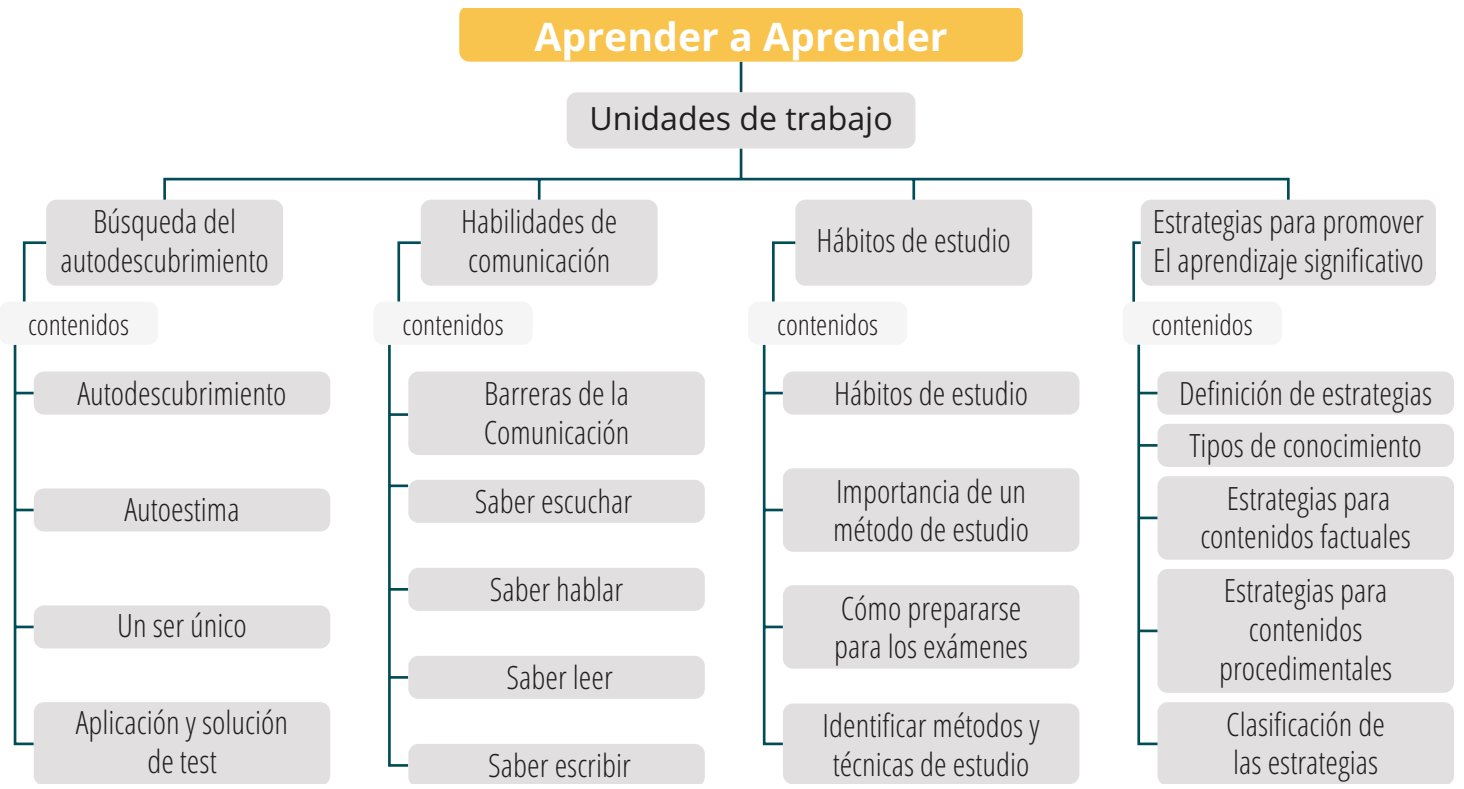

El trabajo desarrollado en el Instituto de Ciencias básicas e Ingeniería (ICBI) se consideró innovador al desarrollar e integrar diferentes estrategias y materiales cuyos resultados se vieron reflejados en el desempeño y aprovechamiento de los estudiantes permitieron que la academia institucional de este instituto generará la propuesta de la nueva versión para el 2017.

Entre las estrategias desarrolladas se encuentran las siguientes:

\section{Perfil de ingreso de los estudiantes.}

Es un informe que presenta los aspectos más relevantes de los estudiantes que recién ingresan con la finali-

dad de ser presentados tanto a los alumnos como a los docentes para tomar acciones e implementar estrategias que les permitan tener un mejor desempeño académico, este perfil es elaborado por cada docente de aprender a aprender y se conforma de los siguientes elementos:

\section{a) Resultados del examen de ingreso.}

A partir de los resultados del examen nacional de ingreso a licenciatura (CENEVAL) la coordinación de docencia del instituto los envía a los docentes de la asignatura para su análisis, de acuerdo al semáforo de aprovechamiento que maneja CENEVAL desde gris con el nivel más BAJO hasta el verde que es MUY ALTO se realiza una tabla de ubicación para cada estudiante: 
Ilustración 5» Resultados del examen de ingreso a licenciatura EXANI(Ceneval) 1er Semestre de licenciatura del ICBI.

\begin{tabular}{c|c|c|c|}
\hline \hline IPMA & \multicolumn{1}{|c}{ IPAN } & \multicolumn{1}{l}{ ICC_EL } & ICC_CL \\
\hline 940 & 844 & 892 & 844 \\
\hline 1228 & 1132 & 916 & 964 \\
\hline 988 & 964 & 1012 & 1012 \\
\hline 1132 & 1060 & 1156 & 1012 \\
\hline 988 & 1036 & 964 & 1036 \\
\hline 1108 & 1180 & 1060 & 1060 \\
\hline 1084 & 1060 & 1156 & 1084 \\
\hline 940 & 1012 & 964 & 1108 \\
\hline 1012 & 1108 & 988 & 1108 \\
\hline 1108 & 1132 & 1012 & 1108 \\
\hline 1132 & 1108 & 1036 & 1108 \\
\hline 1156 & 1156 & 1060 & 1108 \\
\hline 1252 & 1228 & 1252 & 1180 \\
\hline 1156 & 1228 & 1180 & 1228 \\
\hline \hline
\end{tabular}

RESULTADO DEL CENEVAL

\begin{tabular}{ccc}
\hline \hline COLOR & CALIFICACIÓN & $\begin{array}{c}\text { RANGO } \\
\text { CENEVAL }\end{array}$ \\
\hline & $0.0-5.9$ & $700-1059$ \\
\hline & $6.0-6.9$ & $1060-1119$ \\
\hline & $7.0-7.9$ & $1120-1179$ \\
\hline & $8.0-8.9$ & $1180-1239$ \\
\hline & $9.0-10.0$ & $1240-1300$ \\
\hline
\end{tabular}

\begin{tabular}{cc}
\hline \hline IPMA & Pensamiento Matemático \\
\hline IPAN & Pensamiento Analítico \\
\hline ICC_EL & $\begin{array}{c}\text { Estructura de la Lengua v } \\
\text { (Inglés) }\end{array}$ \\
\hline ICC_CL & Comprensión Lectora \\
\hline \hline
\end{tabular}

En la figura anterior se muestran las competencias evaluadas que son:

- Pensamiento matemático (IPMA),

- Pensamiento analítico (IPAN),

- Estructura de la lengua (ICC_EL),

- Comprensión lectora (ICC_CL),

En la tabla las filas representan a cada estudiante con la puntuación y colorimetría obtenida, dichos resultados se muestran en la Academia Horizontal donde se reúnen los docentes que imparten las asignaturas de primer semestre y se identifican las estrategias que se considerarán para el desarrollo de las clases. Entre la cuales se encuentra la preparación de un curso de homologación, canalizar a los alumnos al área de psicopedagogía para reforzar aquellas competencias en que resultaron deficientes.

\section{b) Resultados del Test de Estilos de Aprendizaje}

Considerando el instrumento elaborado por Honey Alonso basado en el modelo Kolb se identifican los estilos de aprendizaje desarrollados por los estudiantes los cuales lo contestan en línea cuyo material fue desarrollado por la Academia institucional de aprender a aprender del ICBI. Anexo 1.

Ilustración 6» Resultados del Test Honey Alonso de Estilos de aprendizaje.

\begin{tabular}{|c|c|c|c|}
\hline & ETILOS DE & APRENDIZAJE & \\
\hline $\begin{array}{l}\text { Cada fil } \\
\text { No se regis }\end{array}$ & $\begin{array}{l}\text { muestra e } \\
\text { ran alumn }\end{array}$ & $\begin{array}{l}\text { registro de cac } \\
\text { os para progran }\end{array}$ & $\begin{array}{l}\text { da alumno } \\
\text { na PAMDIE }\end{array}$ \\
\hline ACTIVO & TEÓRICO & PRAGMÁTICO & REFLEXIVO \\
\hline Muy Alta & Alta & Alta & Alta \\
\hline Alta & Alta & Muy Alta & Baja \\
\hline Moderada & Alta & Alta & Moderada \\
\hline Muy Alta & Alta & Alta & Moderada \\
\hline Moderada & Baja & Baja & Baja \\
\hline Baja & Moderada & Moderada & Moderada \\
\hline Alta & Muy Alta & Muy Alta & Baja \\
\hline Muy Alta & Muy Alta & Alta & Moderada \\
\hline Muy Baja & Muy Alta & Moderada & Moderada \\
\hline Muy Alta & Alta & Alta & Moderada \\
\hline
\end{tabular}

Este test muestra resultados para cuatro estilos: ACTIVO, REFLEXIVO, PRAGMÁTICO Y TEÓRICO en escalas: Muy bajo, Bajo, Medio, Alto y Muy Alto y muestra las preferencias que tienen los estudiantes para enfrentar diferentes ambientes de aprendizaje, los cuales son flexibles y pueden cambiar con el tiempo dependiendo de las estrategias y acciones que se desarrollen. (Sánchez \& Andrade, 2014). Este instrumento también se encuentra de manera digital en repositorio de la universidad en el material de ¿Cómo aprendo, cómo estudio? 
llustración 7» Interpretación de estilo de aprendizaje Activo para 1er Sem de licenciatura del ICBI.

\begin{tabular}{ccc}
\hline \hline \multicolumn{3}{c}{ ESTILOS DE APRENDIZAJE } \\
\hline \hline \multicolumn{3}{c}{ ACTIVO } \\
\hline TENDENCIA & No. ALUMNOS & PORCENTAJE \\
\hline Muy Baja & 1 & $11 \%$ \\
\hline Baja & 1 & $11 \%$ \\
\hline Moderada & 2 & $22 \%$ \\
\hline Alta & 2 & $22 \%$ \\
\hline Muy Alta & 3 & $33 \%$ \\
\hline \hline & \multicolumn{2}{c}{ El grupo presenta un alto índice en la tendencia } \\
MoDERADA con el 22\% y MUY ALTA y ALTA en \\
ACTIVO con un 33\% y 22\% lo cual presenta un \\
grupo dinámico con facilidad de liderazgo, Inquie- \\
tos de impulsivos en caso de ser poco reflexivos.
\end{tabular}

Cada estilo se muestra de manera separada y se brinda un porcentaje de las tendencias y las características de cada una.

\section{c) Resultados del Test de Inteligencias múltiples}

Se desarrolla a partir de la Teoría de las inteligencias múltiples de Howard Gardner, en la cual se conceptualiza la inteligencia como la capacidad de resolver problemas de acuerdo al entorno y las personas pueden desarrollarlas en el transcurso de su vida. Se utiliza la misma colorimetría que en el caso de Ceneval siendo el Gris: Muy Bajo, Rojo: Bajo, Café: Bueno, Amarillo: Muy bueno, Verde: Sobresaliente.

Los resultados de este test se comparan con el de Ceneval principalmente con las competencias de pensamiento matemático y pensamiento analítico y se canalizan a aquellos estudiantes que requieren apoyo pedagógico y asesorías disciplinares.

|lustración 8» Resultados del test de inteligencias múltiples de 1er Sem de licenciatura del ICBI.

\begin{tabular}{|c|c|c|c|c|c|}
\hline \multicolumn{6}{|c|}{ INTELIGENCIAS MÚLTIPLES } \\
\hline $\begin{array}{l}\text { INTELIGENCIA } \\
\text { LINGÜÍSTICA }\end{array}$ & PORCENTAJE & ALUMNOS & $\begin{array}{l}\text { INTELIGENCIA } \\
\text { LÓGICAA } \\
\text { MATEMÁTICA }\end{array}$ & PORCENTAJE & ALUMNOS \\
\hline Muy Bajo & $67 \%$ & 4 & Muy Bajo & $0 \%$ & 0 \\
\hline Bajo & $0 \%$ & 0 & Bajo & $33 \%$ & 2 \\
\hline Bueno & $33 \%$ & 2 & Bueno & $0 \%$ & 0 \\
\hline Muy Bueno & $0 \%$ & 0 & Muy Bueno & $33 \%$ & 2 \\
\hline Sobresaliente & $0 \%$ & 0 & Sobresaliente & $33 \%$ & 2 \\
\hline $\begin{array}{l}\text { INTELIGENCIA } \\
\text { INTERPERSONAL }\end{array}$ & PORCENTAJE & ALUMNOS & $\begin{array}{l}\text { INTELIGENCIA } \\
\text { INTRAPERSONAL }\end{array}$ & PORCENTAJE & ALUMNOS \\
\hline Muy Bajo & $50 \%$ & 3 & Muy Bajo & $50 \%$ & 3 \\
\hline Bajo & $17 \%$ & 1 & Bajo & $17 \%$ & 1 \\
\hline Bueno & $33 \%$ & 2 & Bueno & $0 \%$ & 0 \\
\hline Muy Bueno & $0 \%$ & 0 & Muy Bueno & $33 \%$ & 2 \\
\hline Sobresaliente & $0 \%$ & 0 & Sobresaliente & $0 \%$ & 0 \\
\hline \multicolumn{3}{|c|}{$\begin{array}{l}\text { - Inteligencia Lingüística. El } 67 \% \text { requieren de } \\
\text { habilidades de expresión oral. } \\
\text { - Inteligencia Lógica Matemática. El 33\% } \\
\text { muestra buen dominio en dicha inteligencia. }\end{array}$} & \multicolumn{3}{|c|}{$\begin{array}{l}\text { - Inteligencia Interpersonal. El } 67 \% \text { muestran } \\
\text { pocas habilidades para relacionarse con los } \\
\text { demás y trabajar en equipo. } \\
\text { - Inteligencia Intrapersonal. El } 67 \% \text { tienen } \\
\text { niveles bajos y requieren mejor control de } \\
\text { emociones. }\end{array}$} \\
\hline
\end{tabular}

\section{d) Cuestionario de Hábitos de estudio}

Este instrumento forma parte del material didáctico que se implementó en el material didáctico ya mencionado anteriormente y permite identificar los aspectos que los estudiantes al llegar a la universidad han desarrollado:

- Distribución de tiempo para el estudio

- Motivación para el estudio

- Organización para el estudio 
- Como preparar las notas de clase para estudiar

La información recabada permite diagnosticar a aquellos alumnos a los que les falta desarrollar hábitos de estudio los cuales se abordan en la tercera unidad y a quienes tengan resultados muy bajos, para canalizarlos al área de psicopedagogía.

Ilustración 9» Resultados del Cuestionario de Hábitos de estudio de 1er Sem de licenciatura del ICBI

\begin{tabular}{|c|c|c|c|c|c|}
\hline \multicolumn{6}{|c|}{ HÁBITOS DE ESTUDIO } \\
\hline $\begin{array}{l}\text { PUNTUACIÓN } \\
\text { DISTRIBUCIÓN } \\
\text { DE TIEMPO }\end{array}$ & PORCENTAJE & ALUMNOS & $\begin{array}{c}\text { MOTIVACIÓN } \\
\text { PARA EL ESTUDIO }\end{array}$ & PORCENTAJE & ALUMNOS \\
\hline Muy Bajo & $50 \%$ & 4 & Muy Bajo & $63 \%$ & 5 \\
\hline Bajo & $25 \%$ & 2 & Bajo & $38 \%$ & 3 \\
\hline Bueno & $13 \%$ & 1 & Bueno & $0 \%$ & 0 \\
\hline Muy Bueno & $13 \%$ & 1 & Muy Bueno & $0 \%$ & 0 \\
\hline Sobresaliente & $0 \%$ & 0 & Sobresaliente & $0 \%$ & 0 \\
\hline $\begin{array}{l}\text { ORGANIZACIÓN } \\
\text { PARA EL ESTUDIO }\end{array}$ & PORCENTAJE & ALUMNOS & $\begin{array}{c}\text { COMO PREPARAR } \\
\text { LAS NOTAS DE CLASE } \\
\text { PARA ESTUDIAR } \\
\end{array}$ & PORCENTAJE & ALUMNOS \\
\hline Muy Bajo & $63 \%$ & 5 & Muy Bajo & $38 \%$ & 3 \\
\hline Bajo & $38 \%$ & 3 & Bajo & $50 \%$ & 4 \\
\hline Bueno & $0 \%$ & 0 & Bueno & $0 \%$ & 0 \\
\hline Muy Bueno & $0 \%$ & 0 & Muy Bueno & $13 \%$ & 1 \\
\hline Sobresaliente & $0 \%$ & 0 & Sobresaliente & $0 \%$ & 0 \\
\hline \multicolumn{3}{|c|}{$\begin{array}{l}\text { - En hábitos de estudio se deben poner atención en } \\
\text { distribución de tiempo con un } \mathbf{7 5 \%} \text { de niveles } \\
\text { bajos, } \mathbf{1 0 0 \%} \text { tienen diferencias en organización } \\
\text { para el estudio y el } \mathbf{8 8 \%} \text { no saben preparar } \\
\text { notas para el examen, además en motivación }\end{array}$} & \multicolumn{3}{|c|}{$\begin{array}{l}\text { para el estudio el } 100 \% \text { carecen de éste hábito. } \\
\text { Por lo que es muy importante desarrollar } \\
\text { hábitos de estudio en cada asignatura y en } \\
\text { cada clase. }\end{array}$} \\
\hline
\end{tabular}

\section{e) Encuesta socioeconómica.}

Finalmente, una semana antes de iniciar las clases se lleva a cabo un curso de inducción, se realiza una encuesta socioeconómica que permite complementar la información anterior y reforzar las estrategias, entre los datos solicitados se encuentran:

- Lugar de procedencia

- Si vivirán solos por primera vez

- Si la licenciatura elegida fue su primera opción de carrera

- En caso negativo ¿Cuál fue su primera opción? Y ¿por qué no pudo acceder a ella?

llustración 10» Datos que conforman la Encuesta Socioeconómica para perfil del estudiante. ICBI.

\begin{tabular}{c}
\hline \hline DATOS GENERALES \\
\hline Edad promedio de los alumnos 19 años. \\
\hline • Eluctúan entre 18 y 32 años. \\
\hline \hline
\end{tabular}

Ilustración 11» Datos que conforman la Encuesta Socioeconómica para perfil del estudiante. ICBI.

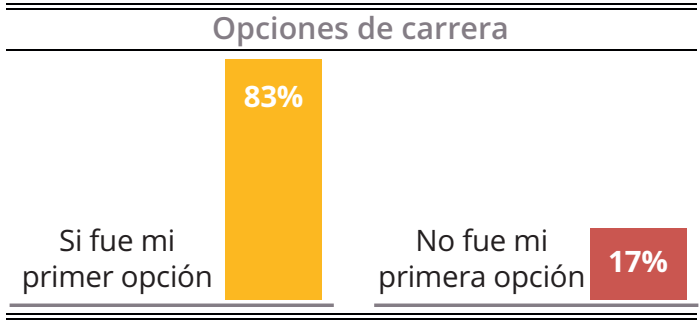

El perfil del estudiante permite conocer de inicio aspectos relacionados con el desempeño académico que los recién egresados muestran, aquellos que presentan niveles muy bajos y que se consideran en riesgo de deserción o de reprobación se canalizan al área de psicopedagogía del ICBI, para pertenecer al Programa de Actividades para el desempeño integral del Estudiante (PAMDIE) el cual también es único en la institución y fue implementado desde el semestre julio - diciembre del 2013, presentando cada final de curso los resultados ante las autoridades del instituto. 
La participación de los docentes de aprender a aprender es relevante ya que dan seguimiento tanto a los estudiantes que pertenecen a PAMDIE, como al grupo en general a través de los contenidos del curso, cuyas actividades y estrategias se continúan describiendo por cada unidad temática.

\section{Desarrollando al estudiante estratégico}

\section{a) Esquema de análisis situacional.}

Este instrumento se presenta a través del taller "El Estudiante Estratégico" y tiene como finalidad que comprendan e integren los elementos de la planeación estratégica a través de plantear su proyecto de vida en la dimensión profesional principalmente, el esquema contiene el Plan estratégico y Plan táctico:
Ilustración 12» Esquema de Análisis situacional para el Estudiante Estratégico. Aprender a Aprender. ICBI-UAEH.

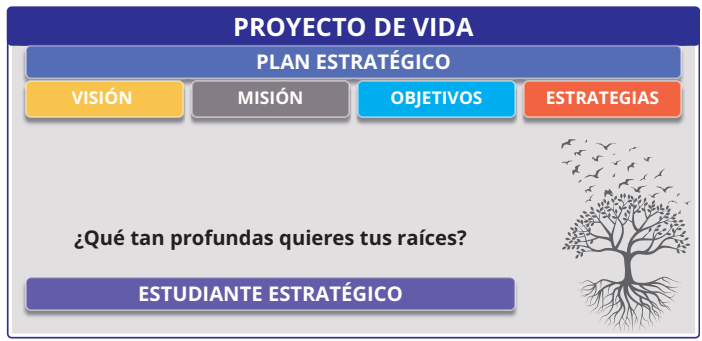

El Plan Estratégico pretende que los estudiantes puedan proyectar y plantear su Visión a los próximos 5 años (ya estando egresados), en la Misión determinarán el rumbo en el presente para conseguir la Visión, lo cual se logrará a través de diseñar los objetivos durante el curso de su carrera profesional, dónde, se les brindarán las Estrategias de Aprendizaje las cuales se pondrán en marcha con la ejecución del Plan Táctico a fin de ir alcanzando las metas a corto, mediano y largo plazo.

Ilustración 13» Estrategias de Aprendizaje del Plan estratégico en el Esquema de Análsis situacional.

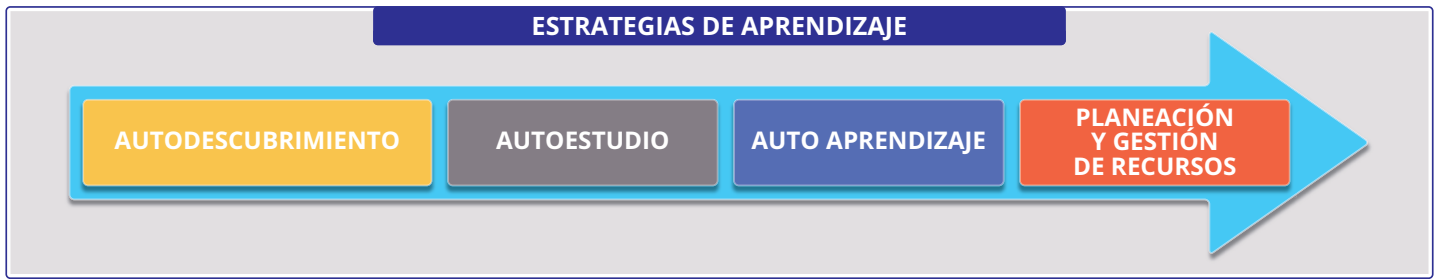

La ejecución del plan táctico permitirá poner en operación y alcanzar el Plan Estratégico, se redactan las metas como aquellos indicadores de índole cuantitativo donde se especifican claramente las acciones y actividades que se llevarán a cabo, así como los resultados que se esperan a corto, mediano y largo plazo los que podrán ser medidos y comparados para poder valorar el impacto y logro de las metas antes citadas.

Este plan táctico se estructura con tres componentes que son la Conciencia Situacional; es un análisis del contexto y situación en la que se ubica al llegar a la institución el alumno aceptado en la licenciatura, debe recopilar, indagar e investigar todo lo relacionado con la universidad, las autoridades y lugares esenciales, involucra a los docentes, investigadores, personal académico y administrativo, para ello agenda entrevistas, realiza vídeos de recorridos institucionales y finalmente realiza un análisis FODA para tener claro cuáles serán las accio- nes y actividades para mejorar su desempeño personal y académico.

Ilustración 14» Plan táctico del Esquema de Análisis situacional para el Estudiante Estratégico. Aprender a Aprender ICBI- UAEH.

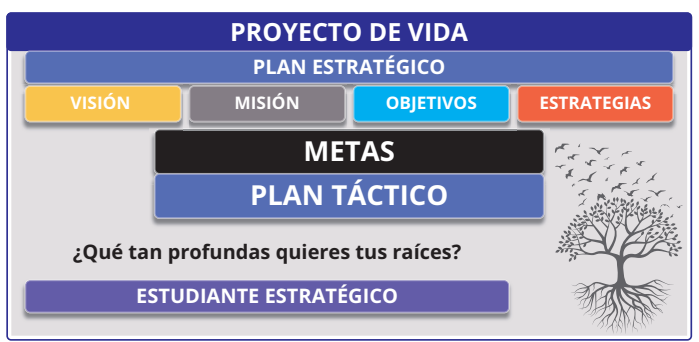

La Conciencia Situacional también involucra el autoconocimiento y comprensión intrapersonal para identificar fortalezas y debilidades, cuyo instrumento es la autobiografía, ésta permite desarrollar de manera clara el esquema de análisis situacional para consultar, comparar y retroalimentar el desempeño plasmado en las 
metas, además los resultados se consideran en el Perfil del Estudiante mencionado anteriormente.

Ilustración 15» Plan táctico del Esquema de Análisis situacional para el Estudiante Estratégico. Aprender a Aprender. ICBI.UAEH.

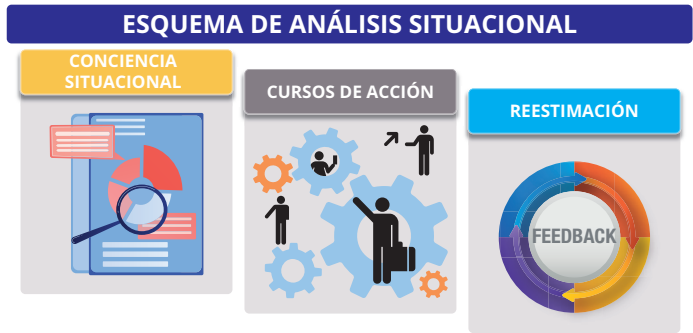

El Curso de Acción, como su nombre lo indica, permite poner en marcha acciones tendientes al logro de sus objetivos y ejecutar las estrategias de aprendizaje establecidas en Plan Estratégico mediante los contenidos y actividades plasmadas en el curso a través de la cuatro unidades temáticas:

Ilustración 16» Curso de Acciones del Plan táctico del Esquema de análisis situacional. Aprender a Aprender. ICBI

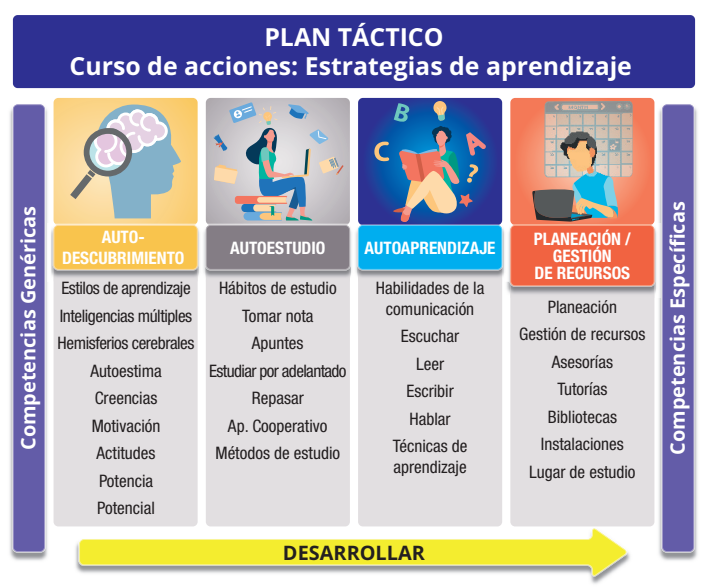

Estrategia de aprendizaje de autodescubrimiento. Permite un análisis intrapersonal que facilite el punto de partida de cada estudiante, para ello realizan cuestionarios y test, además de actividades para fortalecer sus creencias, motivación, autoestima, actitudes, aptitudes y valores además de conocer y desarrollar su potencial.

Estrategia de Aprendizaje de Autoestudio. El desarrollo de hábitos de estudio cuyos resultados figuran como producto del cuestionario previamente realizado muestra desde 2013 que se implementó esta asignatura que es una de las principales carencias mostradas por los estudiantes que ingresan al nivel licenciatura y de los factores principales asociados a la reprobación y deserción, por lo que, se trabaja de manera intensa en esta estrategia, los hábitos que principalmente tienen prioridad en el primer semestre son:

\section{Ilustración 17» Hábitos del Estudiante Estratégico.-} Aprender a Aprender. ICBI-UAEH.

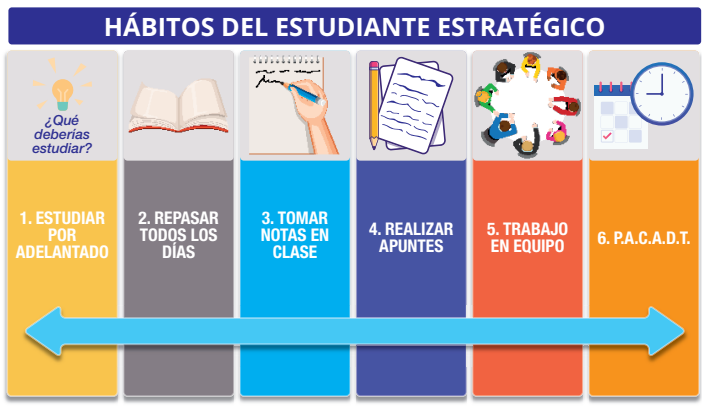

- Estudiar por adelantado.

- Repasar las clases todos los días.

- Tomar notas en clase.

- Realizar apuntes en casa.

- Trabajar en equipo. Estrategia de aprendizaje cooperativo.

- Planeación de actividades y administración del tiempo.

El desarrollo de los hábitos anteriores es obligatorio durante el primer semestre para lo que se solicita el apoyo a través de los directivos de la institución para que los docentes le den seguimiento durante el semestre y también se da a conocer a los de segundo semestre, aunque la implementación y seguimiento en semestres posteriores no ha sido exitosa del todo.

Estrategia de Autoaprendizaje. Con esta estrategia se desarrollan las habilidades de la comunicación siguientes: saber escuchar, saber leer, saber escribir, saber hablar, así como el manejo y diseño de técnicas de aprendizaje tales como: mapas conceptuales, mapas mentales, cuadros sinópticos, ensayos, resumen, para esta estrategia se cuenta con material multimedia elaborado por la Academia de Aprender a Aprender del ICBI, para desarrollar técnicas de lectura y diseño de mapas conceptuales a partir de los lineamientos de su creador Joseph Novak. (Anexo 2). 
Estrategia de Planeación y Gestión de Recursos. El desarrollo de la neurodidáctica y en particular el estudio de la corteza prefrontal permite identificar como un área relevante el de las funciones ejecutivas o de orden superior como la planeación, toma de decisiones, ética, visión a futuro, entre otras, las cuales alcanzan su máximo desarrollo a partir de los 21 años y en algunos casos en los hombres puede ser después de esa edad, por lo que ha resultado un fracaso para los docentes suponer que los estudiantes al ingresar a la universidad son capaces de planear su propio proyecto de vida, cuando la maduración de muchos de ellos no ha alcanzado su máximo potencial, por lo que esta estrategia pretende fortalecer el desarrollo de estas funciones ejecutivas.

El último componente del Plan táctico es la Reestimación la cual se aplica en cada corte de evaluación institucional que se compone de tres exámenes parciales y estos resultados se comparan con las metas establecidas en el Plan Estratégico, se consideran nuevas rutas de acción y se entrega un informe al docente de Aprender a Aprender en el que se establece con el estudiante, el compromiso de llevar a cabo el nuevo plan para mejorar el desempeño académico y ser canalizado en caso necesario al área de psicopedagogía.

\section{Encuentro de habilidades de la comunicación.}

Otra actividad que ha significado un cambio e innovación con la implementación de la Asignatura de Aprender a Aprender es la organización y puesta en marcha del Encuentro de habilidades de la comunicación, mismo que dio inicio en el año 2013 con el 1er evento llevado a cabo mediante convocatoria abierta para todos los estudiantes de primer semestre. Los resultados del test de inteligencias múltiples muestran que la inteligencia lingüística en los estudiantes del ICBI tiene porcentajes por debajo de la media y es la misma situación en la competencia de comprensión lectora del examen de ingreso a licenciatura EXANI, lo cual justifica y fortalece el desarrollo de este tipo de actividades.
Ilustración 18» Encuentro de Habilidades de la comunicación. ICBI. UAEH.

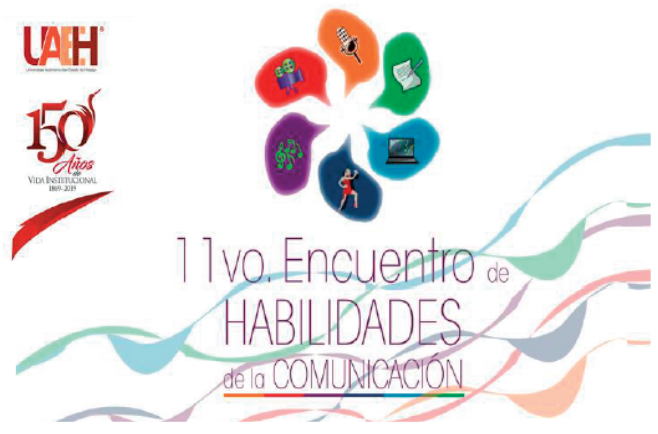

La finalidad es desarrollar las habilidades de la comunicación, creatividad, innovación y liderazgo a través de diferentes modalidades en donde se eligen las mejores propuestas y participaciones, realizando una ceremonia de premiación por parte de las autoridades. Las modalidades que conforman el encuentro son:

- Conferencia.

- Debate.

- Juego didáctico.

- Comprensión lectora.

La conferencia debe llevarse a cabo con el apoyo de docentes expertos en el área que el estudiante elija cuyos temas deben ser relacionados con su licenciatura y deben presentar alguna innovación, tendencia o recorrido histórico de la disciplina, los lineamientos de participación son rigurosos y deben realizarse ensayos previos, así como revisiones del material para presentar, además de asistir a un taller sobre expresión oral.

Ilustración 19» Exposición de conferencia en el 110 Encuentro de Habilidades de la Comunicación. 1er Semestre. Ing Industrial.ICBI.

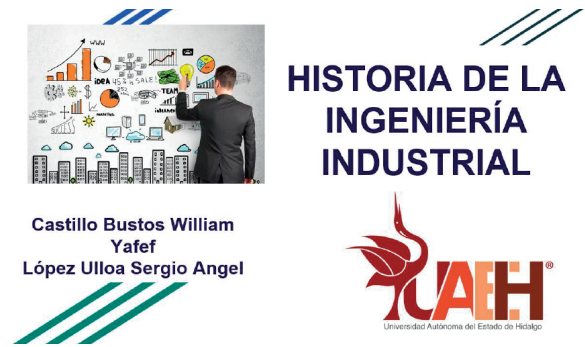


El debate es otra técnica de comunicación oral grupal que permite desarrollar diferentes habilidades aparte de la expresión oral, como; la habilidad de argumentación en donde se debe defender una postura ante una problemática de orden social que genera una controversia, los participantes deben investigar y contar con fuentes confiables y actaulizadas sobre las temáticas presentadas en la convocatoria, se presentan en equipos de máximo 6 personas y ante un jurado, y con un tiempo delimitado para cada participación deberán argumentar y defender su postura para que al final de las tres fases: argumentación, réplica, contrarréplica y conclusiones, se elige al equipo ganador que pasará a la siguiente ronda hasta llegar a la fase final donde se enfrentan los dos equipo ganadores de todas las rondas previas ante un público numeroso.

Ilustración 20» Final del evento de Debate del Encuentro de Habilidades de la comunicación.-ICBI.

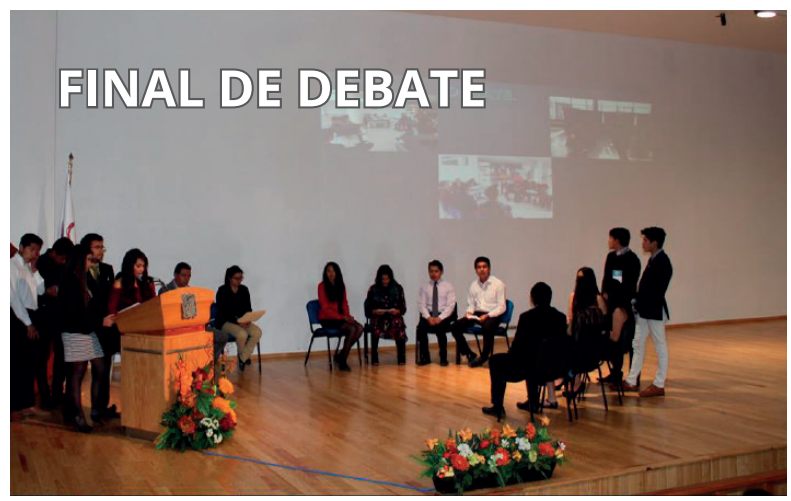

El juego didáctico es otra modalidad de participación, consiste en diseñar material lúdico que facilite el aprendizaje de las asignaturas del semestre, dicho juego debe contar también con la asesoría de los expertos de la disciplina ya que los lineamientos para participar son rigurosos en cuanto la rúbrica de evaluación donde los materiales de elaboración, la creatividad, innovación y nivel de complejidad son los principales aspectos para determinar al ganador de dicha modalidad.

Comprensión lectora es la última modalidad de participación, tiene como finalidad desarrollar las técnicas de identificación de ideas principales y secundarias de un texto después de realizar la lectura de comprensión, además se complementa con el diseño de un organizador gráfico donde muestran el nivel de análisis y síntesis, contestando al final un cuestionario de comprensión del material, se puede participar en parejas y las lecturas son de carácter científico y actualizadas.

Ilustración 21» Evento de Juegos didácticos del 110 Encuentro de Habilidades de la Comunicación. 1er Semestre. ICBI.

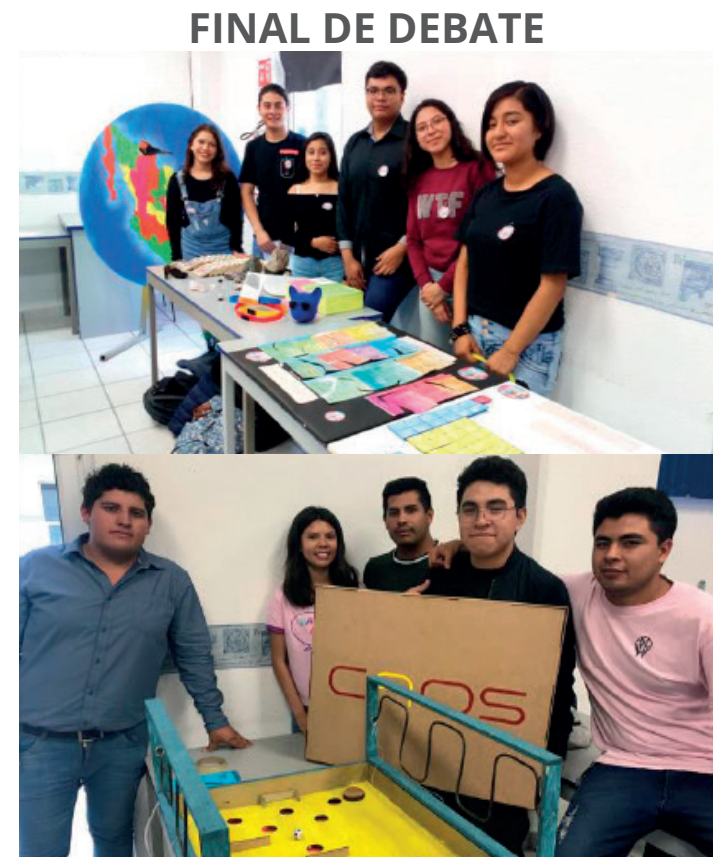

Ilustración 22» Evento de comprensión lectora del 110 Encuentro de Habilidades de la Comunicación. ICBI.

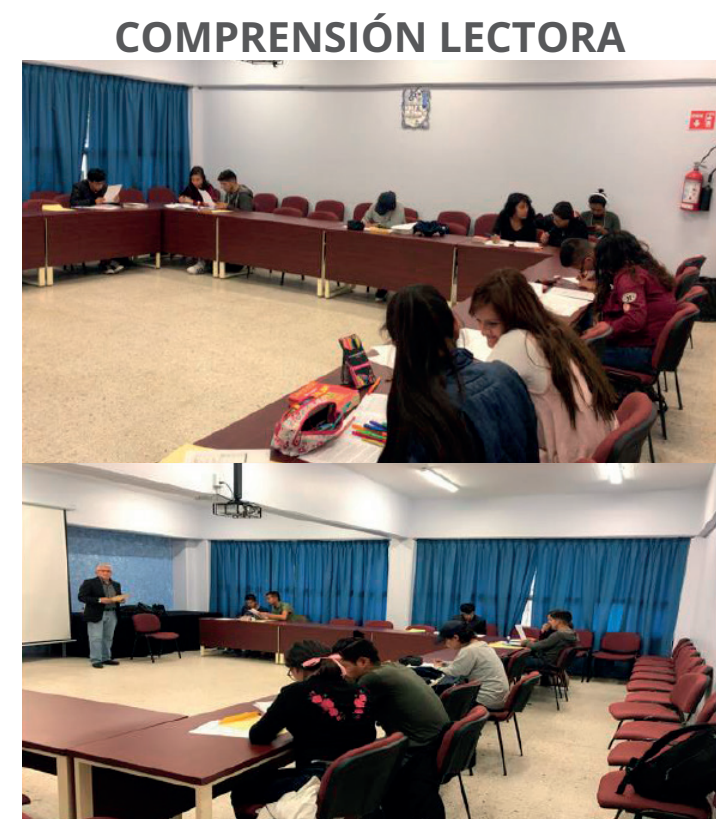


Las actividades planteadas son parte del trabajo que se desarrolla en el Instituto de Ciencias básicas e Ingeniería (ICBI) por los docentes que conforman la academia institucional de Aprender a Aprender y como ya se mencionó se realizan solamente en el mencionado plantel, los resultados de dichos trabajo se dieron a conocer a través de distintos medios como: el periódico, radio y publicaciones institucionales por lo que dicha academia fue convocada para llevar a cabo la actualización de la asignatura y generar la versión 2018, la nueva versión se presentó ante las autoridades de la universidad siendo aprobada e implementada en el campus Tizayuca, esta nueva actualización cuenta con la estructura siguiente:

1 Estudiante estratégico.

2 Autodescubrimiento ¡Impulso para el aprendizaje!

3 Ejercitando mi cerebro; ¡En acción para la resolución de problemas!

4 Comunicación y aprendizaje, ¡Desarrollando habilidades!!

5 Mi botiquín del aprendizaje: Técnicas y métodos de estudio

Esta propuesta contiene las actividades desarrolladas en el instituto con la versión inicial, además de incluir el bloque para el desarrollo de habilidades de autorregulación, metacognición, habilidades del pensamiento que incluye la capacitación docente en neurodidáctica para adecuar las estrategias de enseñanza a las bases neurológicas del aprendizaje, la memoria, el pensamiento así como la gestión de las emociones.

\section{CONCLUSIONES}

$\mathbf{E}$ ste material representa el trabajo colegiado de los docentes de Aprender a Aprender que inicia en el semestre enero - junio 2013 cuando se implementa la Reforma Educativa de nivel de licenciatura en la UAEH, específicamente en el ICBI, esta asignatura cuyo antecedente es la de Técnicas de enseñanza y aprendizaje que se impartía en la Licenciatura de Ingeniería Minero Metalúrgico ofrece el apoyo psicopedagógico que no se tenía considerado para los alumnos de reciente ingreso a la universidad, no solo se estructura de contenidos, sino de actividades, talleres y un Encuentro de habilidades de la comunicación.
Aprender a Aprender es una asignatura que a pesar de su poca aceptación y perspectivas bajas de éxito actualmente formará parte de los siguientes rediseños y planes de estudio de los programas educativos de la institución, el ICBI es el instituto pionero que a través de sus autoridades brindó el apoyo y confianza a la academia de Aprender a Aprender e incluso da pauta para la creación del área de psicopedagogía y la implementación del PAMDIE, como apoyo para la formación integral de los estudiantes que son la esencia de toda institución educativa.

\section{BIBLIOGRAFÍA}

Araoz Robles, Edith (2008) Estrategias para aprender a aprender; Reconstrucción del conocimiento a partir de la lectoescritura, México: Pearson.

Argudín, Y., \& Luna, M. (2016). Atrévete a pensar, desarrollo del pensamiento crítico por medio de la lectura crítica. México: Trillas.

Bilbao, M. d., \& Velasco, P. (2014). Aprendizaje con inteligencias múltiples: cómo identificar las inteligencias múltiples, cómo desarrollarlas y cómo evaluarlas. México: Trillas.

Cuevas, L. T. (2010). Modelo Curricular: Reto de las políticas de innovación educativa para la gestión de la reforma universitaria. Pachuca Hgo: Praxis.

Fonseca, S., Cortés, A., Pineda, M. I., \& Lemus, F. (2011). Comunicación oral y escrita. México: Pearson.

García, B., \& García, B. (2016). Coaching práctico en educación. España: Dextra.S.L.

González, R., González, J. A., \& Rodríguez, S. (2005). Estrategias y técnicas de estudio. España: Pearson Education, S. A.

Hernández Rojas , G., \& Díaz Barriga Arceo, F. (2010). Estrategias docentes para un aprendizaje significativas Una interpretación constructivista y Gerardo Hernández Rojas Año 1998 - 2010 Unidad 6. México: McGRAW-HILL.

Huerta, M. (2016). La estrategia en el aprendizaje. México: Neisa.

Logatt, C., Rosler, R., Labath, L., \& Guaita, V. (Mayo de 2017). Capacitación docente en Neurociencias. [Material de curso]. Argentina: Asociación Educar 
para el desarrollo humano.

Nieto, J. (2011). Neurodidáctica. Madrid: CCS.

Rangel, M. (2016). El debate y la argumentación, teoría, técnica y estrategias. México: Trillas.

\section{RECURSOS MULTIMEDIA:}

Escamilla, C., Ortega, J., Polo, C. \& Straffon, O. (2015). ¿Cómo aprendo? Y ¿Cómo estudio? [Multimedio educativo] Recuperado de: http://ceca.uaeh.edu. mx/comoaprendo/ el 20 octubre del 2017.

Ortega, J., Polo, C. \& Straffon, O. (2017). Técnicas de aprendizaje. Mapa Conceptual.[Multimedio educativo] Recuperado de: http://ceca.uaeh.edu.mx/
Salas, R. E. (2014). Estilos de aprendizaje a la luz de la neurociencia. México: Magisterio.

Sánchez, L., \& Andrade, R. (2014). Inteligencias múltiples y estilos de aprendizaje ISBN:978-607-622-041-2. México: Alfaomega.

tecnics_aprendizaje/index.html Recuperado el 20 octubre del 2017.

Ortega, J., Polo, C. (2017). Técnicas básicas de aprendizaje para el análisis de textos.[Multimedio educativo] Recuperado de: http://ceca.uaeh.edu.mx/tecnicas_ basicas/index.html el 20 octubre del 2017

\section{ANEXOS}

ANEXO 1. ¿Cómo aprendo, cómo estudio? Material didáctico para la asignatura de Aprender a Aprender. http://ceca.uaeh.edu.mx/comoaprendo/
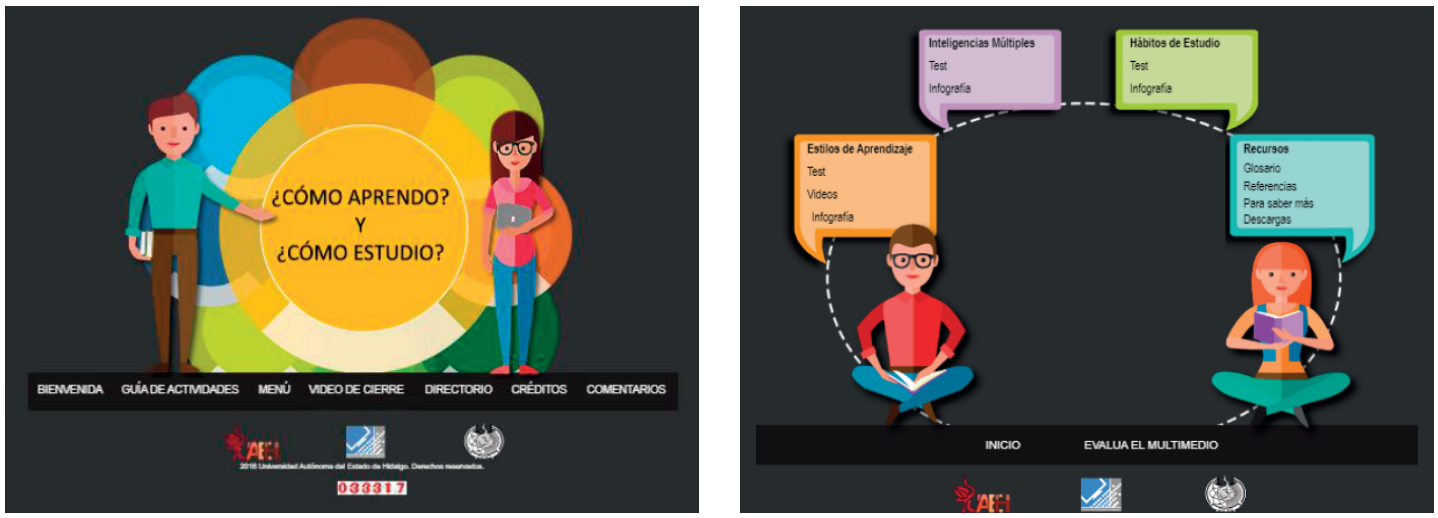

ANEXO 2. Técnicas de aprendizaje. Material didáctico para la asignatura de Aprender a Aprender: http://ceca. uaeh.edu.mx/tecnicas aprendizaje/ 